\title{
Dietary Profile of Rhinopithecus bieti and Its Socioecological Implications
}

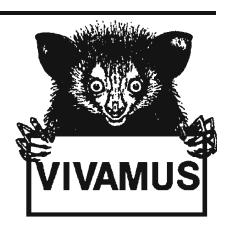

\author{
Cyril C. Grueter • Dayong Li • Baoping Ren • \\ Fuwen Wei • Carel P. van Schaik
}

Received: 26 March 2009 / Accepted: 15 June 2009 / Published online: 14 July 2009

(C) Springer Science + Business Media, LLC 2009

\begin{abstract}
To enhance our understanding of dietary adaptations and socioecological correlates in colobines, we conducted a 20-mo study of a wild group of Rhinopithecus bieti (Yunnan snub-nosed monkeys) in the montane Samage Forest. This forest supports a patchwork of evergreen broadleaved, evergreen coniferous, and mixed deciduous broadleaved/coniferous forest assemblages with a total of 80 tree species in 23 families. The most common plant families by basal area are the predominantly evergreen Pinaceae and Fagaceae, comprising 69\% of the total tree biomass. Previous work has shown that lichens formed a consistent component in the monkeys' diet year-round (67\%), seasonally complemented with fruits and young leaves. Our study showed that although the majority of the diet was provided by 6 plant genera (Acanthopanax, Sorbus, Acer, Fargesia, Pterocarya, and Cornus), the monkeys fed on 94 plant species and on 150 specific food items. The subjects expressed high selectivity for uncommon angiosperm tree species. The average number of plant species used per month was 16 . Dietary diversity varied seasonally, being lowest during the winter and rising dramatically in the spring. The monkeys consumed bamboo shoots in the summer and bamboo leaves throughout the year. The monkeys also foraged on terrestrial herbs and mushrooms, dug up tubers, and
\end{abstract}

C. C. Grueter $(\bowtie) \cdot$ C. P. van Schaik

Anthropological Institute and Museum, University of Zürich, 8057 Zürich, Switzerland

e-mail: cyril_grueter@eva.mpg.de

D. $\mathrm{Li}$

Qinling Golden Snub-nosed Monkey Research Center, College of Life Science, Northwest University, 710069 Xi'an, China

B. Ren · F. Wei

Key Laboratory of Animal Ecology and Conservation Biology, Institute of Zoology, Chinese Academy of Sciences, 100101 Beijing, China

Present Address:

C. C. Grueter

Department of Primatology, Max Planck Institute for Evolutionary Anthropology, 04103 Leipzig, Germany 
consumed the flesh of a mammal (flying squirrel). We also provide a preliminary evaluation of feeding competition in Rhinopithecus bieti and find that the high selectivity for uncommon seasonal plant food items distributed in clumped patches might create the potential for food competition. The finding is corroborated by observations that the subjects occasionally depleted leafy food patches and stayed at a greater distance from neighboring conspecifics while feeding than while resting. Key findings of this work are that Yunnan snub-nosed monkeys have a much more species-rich plant diet than was previously believed and are probably subject to moderate feeding competition.

Keywords diet $\cdot$ China $\cdot$ colobine $\cdot$ feeding ecology $\cdot$ food competition

\section{Introduction}

Researchers study primate food habits for a variety of reasons. Such studies may reveal a species' resource requirements (Litvaitis 2000), and diet-related factors such as nutritional quality, distribution, and spatiotemporal fluctuations in abundance of food may also have far-reaching influences on the emergence of primate social organizations and social structure (Chapman 1990; van Schaik 1989; Wrangham 1980). They may affect group size (Kirkpatrick 1996), tendency toward fissionfusion (Anderson et al. 2002; van Schaik and van Noordwijk 1988), and the intensity and the nature of feeding competition (scramble and contest; Janson and van Schaik 1988; Koenig et al. 1998; Saj and Sicotte 2007a; van Schaik and van Noordwijk 1988). Nonhuman primates exhibit clear preferences for particular food items (Milton 1981). Many factors - both internal and external- mold patterns of food choice in primates, e.g., energy needs, nutrient requirements, constraints of the digestive system, body size, chemical and structural properties of foods, and spatiotemporal availability of food resources (Barton and Whiten 1994; Garber 1987; Kay 1984; Lambert 2007; McKey et al. 1981; Milton 1984; Oates 1987; Oftedal 1991).

Colobines possess specialized capacious and partitioned stomachs (Caton 1998; Stevens 1988) where microbial fermentation of cellulose takes place (Bauchop and Martucci 1968; Hume 1989; Kay and Davies 1994; Kuhn 1964). This adaptation enables them to eat food containing high levels of structural polysaccharides, i.e., cellulose and related compounds. Overall, colobines can be classified as herbivores, ingesting flowers, fruits, leaves, and seeds to varying degrees (Bennett and Davies 1994; Fashing 2007; Kirkpatrick 2007; Oates 1994). Another common feature of colobine feeding ecology is their seasonally varying dietary spectrum as a response to temporal variation in food resource availability, of which switching to less nutritious plant foods (mature leaves) during periods of shortage of preferred food items is a key element (Dasilva 1994; Fashing 2001b; Koenig and Borries 2001; Struhsaker 1975). Although this is true for many tropical-dwelling colobines, some outliers within the Colobinae exemplify the subfamily's great plasticity pertaining to environmental conditions and demonstrate that dietary strategy is only partly determined by evolutionary history (sensu Struhsaker and Oates 1975). A case in point are the Yunnan or black-and-white snub-nosed monkeys (Rhinopithecus bieti) 
of the temperate climate zone, which despite similar anatomy and presumably physiology, exhibit a somewhat different foraging strategy.

Previous work has revealed that Yunnan snub-nosed monkeys are highly dependent on lichens (Kirkpatrick 1996; Xiang et al. 2007), thus being the only anthropoid primate whose main food is not a plant. Their dietary regimen has been shown to vary geographically and to depend on overall habitat condition and productivity: in high latitude/high altitude habitats, lichens constitute the major fraction of the diet in virtually every month and are complemented with leaves from dicots and monocots (Kirkpatrick 1996; Xiang et al. 2007). The natural environment of Rhinopithecus bieti is characterized by striking seasonal variation in food resource availability. Winter is a period of plant food deprivation, and an almost exclusive dependence on carbohydrate-rich lichens has been regarded as a key ecological adaptation (Kirkpatrick 1996; Xiang et al. 2007; Grueter and Xiang 2008). Groups associated with more productive habitats at lower elevations and latitudes have a more species-rich diet and include a substantial proportion of nonlichen foods on a seasonal basis, such as immature leaves, fruits/seeds, buds, flowers, bamboo shoots, and bark of various plants (Ding and Zhao 2004; Yang and Zhao 2001).

We conducted the study in the Samage Forest, which is located in the central part of the geographical range of Rhinopithecus bieti and thus is intermediate in altitude and latitude compared to the populations in the north and south. In a companion paper, we documented seasonality in food use and fallback strategies of Rhinopithecus bieti at Samage (Grueter et al. 2009). We showed that lichens were chosen year-round and comprised ca. $67 \%$ of all the feeding records. Lichens were complemented with plant material, viz. 16\% buds and young leaves, $11 \%$ fruits, $4 \%$ mature leaves, and $2 \%$ other items. Seasonal feeding patterns on plant items exactly matched the temporal variation in the availability of the main plant phenophases. The monkeys exploited immature leaves prolifically in spring and ingested heavy quantities of fruit in summer and fall. Our primary aim here is to document the overall dietary spectrum of Rhinopithecus bieti at Samage and to provide a thorough assessment of habitat composition. The findings are important for comprehending the species' resource requirements and the carrying capacity of the habitat and thus have implications for conservation management.

An additional aim relates to the question of how diet affects socioecology. For colobines, researchers have discussed the issue especially in relation to the importance of scramble competition. Scramble competition is the collective exhaustion of limited resources, leading to lower foraging efficiency for all group members (Janson and van Schaik 1988). Scramble competition increases as groups increase in size and is thought to limit group size for many primates (Janson and Goldsmith 1995). As a result of a more rapid depletion of food patches, larger groups are forced to travel farther to ensure procurement of a sufficient amount of the food (Chapman and Chapman 2000; Janson and van Schaik 1988; Majolo et al. 2008; van Schaik and van Noordwijk 1988). Scramble competition is usually inferred if there is a positive association between group size and home range size or day journey length. Based on weak relationships between these variables in combination with small group sizes and ubiquity of food resources, folivorous or frugivorous-folivorous primates such as colobines have traditionally been viewed as experiencing only a low intensity of intragroup scramble competition (Fashing 
2001a; Isbell 1991; Janson and Goldsmith 1995; Sterck et al. 1997; Yeager and Kirkpatrick 1998; Yeager and Kool 2000). However, group size effects have recently been demonstrated among various folivores (Gillespie and Chapman 2001; Koenig et al. 2008; Majolo et al. 2008; Saj and Sicotte 2007b; Snaith and Chapman 2008; Teichroeb et al. 2003; cf. Steenbeek and van Schaik 2001).

This study of a colobine living in very large groups may help to shed some more light on the issue. Rhinopithecus bieti are also notable for having an unusual social organization: they live in large bands that are composed of distinct core one-male units (OMU). Given the fact that lichens occur in profusion in their natural habitat, at least currently, Rhinopithecus bieti are thought to be free to form large groups, and food competition would not be expected to be prevalent (ibid.). The evidence for scramble competition in Rhinopithecus bieti is scant: we have previously shown a positive correlation between group size and home range size, controlling for productivity, for different populations of $R$. bieti, indicating scramble competition effects (Grueter et al. 2008a). However, home range size is probably not as good a proxy for competition as day journey length, the latter correlating less strongly with group size (Grueter and van Schaik, unpub. data). A different picture may emerge when considering nonlichen foods, i.e. plant resources, which are the focus here. We assess the degree to which the lichenivorous-folivorous-frugivorous dietary regimen of Rhinopithecus beiti generates potential for scramble and also contest competition. The tests presented here are rudimentary given the challenges of observing wild Rhinopithecus bieti. If food competition occurs, then we predict that 1) preferred food species (species with high selection ratios) occur at low densities across the home range and are spatially clumped and thus can probably not accommodate all band members (interunit contest; $c f$. Koenig et al. 1998); 2) valued patches of food are being depleted (intraband scramble; $c f$. Snaith and Chapman 2005), and 3) unit members avoid co-feeding (intraunit scramble or contest; Saj and Sicotte 2007b) by having fewer nearest neighbors when feeding versus resting, assuming that dispersion reduces competition ( $c f$. van Schaik and van Noordwijk 1988).

\section{Methods}

\section{Study Site}

We conducted the study in the predominantly temperate Samage Forest near the village of Gehuaqing $\left(27^{\circ} 34^{\prime} \mathrm{N}, 9^{\circ} 17^{\prime} \mathrm{E}\right)$ in Yunnan's Baimaxueshan National Nature Reserve. Narrow valleys and steep hillsides characterize the topography at the site and land cover is a mosaic of mixed coniferous and deciduous-broadleaf forest (at 2900-3600 m), subalpine George's fir forest (3500-4000 m), montane sclerophyllous oak forests (3200-3500 m), subtropical evergreen broadleaf forest (2500-3000 m), Yunnan pine forest $(2500-3100 \mathrm{~m})$, as well as cattle pastures at various elevations. Umbrella bamboos (Fargesia spp.) and rhododendrons formed an important element of the underbush in all vegetation types. Parts of the Samage Forest have been selectively logged, and anthropogenic disturbance in the form of livestock grazing and collection of NTFP (nontimber forest products) is still widespread. The habitat of the monkeys at this locality ranged from $2500 \mathrm{~m}$ to 4000 
$\mathrm{m}$ and included all major vegetation types, with mixed forest being the most used ecotype and clearcuts being unsuitable habitat for Rhinopithecus bieti. The semihabituated focal group comprised $c a$. 410 members.

\section{Climate}

Annual rainfall was $1004 \mathrm{~mm}$, and mean annual temperature was $14.3^{\circ} \mathrm{C}$ at $2448 \mathrm{~m}$ ( $800 \mathrm{~m}$ below the altitude the focal group most frequently visited). Distribution of precipitation was highly irregular, but temperature varied strikingly with seasons: there was a steep increase in rainfall from spring onwards and a prolonged winter drought season with freezing nights (Grueter et al. 2009; Li et al. 2008). Complete snow cover rarely lasted for more than a few days within the frequently used zone of the group, as snowfall was followed by prolonged sunny days.

\section{Data Collection}

C. C. Grueter collected data on diet composition via scan sampling over 20 mo between September and July 2007. On 116 d, we obtained a total of 3872 feeding records: 1151 in fall (September-November), 772 in winter (December-February), 1314 in spring (March-May), and 635 in summer (June-August). The rugged terrain with steep-sided ravines and impenetrable undergrowth (bamboo, etc.) made tracking difficult, and thus distance observations from prominent topographical features (rocks, etc.) with the use of a spotting scope were the methods of choice. Occasionally we also observed the group at close range.

We took scans of all visible individuals at $15 \mathrm{~min}$ or 30 -min intervals. If a large number of monkeys were in view, we chose 30-min scans; if only a small number was visible, we performed 15-min scans. Scans had to be completed $\geq 5$ min before the beginning of the next scan. Every scan included information on date, time, and weather conditions. For every subject scanned, we recorded age, sex, activity, as well as distance and identity (age-sex class) of the nearest neighbor. Scan data were spread more or less evenly throughout the day. We divided age/sex classes into the following categories: adult male, adult female, juvenile ( $c$. 1-4 yr old), subadult male, and infant ( $<1 \mathrm{yr}$ ). We used the category SAMOF (subadult male or female) for cases wherein it was not possible to determine the sex of an individual whose body size was close to or larger than that of an adult female, but was not accompanied by an infant ( $c f$. Bleisch et al. 1993).

Scan records of feeding behavior also included the food item, plant part, and its age as well as plant species whenever possible. We distinguished the following foods: lichens (fruticose vs. foliose), young leaves (including spring buds/shoots), mature leaves, buds (dormant leaf buds), flowers, flower buds, bark, pith, fruit or seeds (both ripe and unripe), invertebrates, snow, fungi, water, bamboo shoots, and tuber. It was usually difficult to see whether the small fruits were eaten wholly or whether the flesh was discarded. If we were unable to identify the tree taxon visually, we attempted to collect some samples from that feeding tree or a nearby tree of the same taxon for later identification.

Outside of scan sessions, we recorded all partially consumed and discarded foods on the forest floor with tooth marks or other signs of having been handled by the 
monkeys. We used evidence from such feeding signs as a complementary measure to estimate seasonal variance in diet composition. We used the diameter of a feeding litter to quantify remains roughly as small ( $<1 \mathrm{~m}$; score 1$)$, medium sized (1-3 m; score 2), or large ( $>3 \mathrm{~m}$; score 3 ). Observational sampling was usually biased toward arboreal feeding, so the importance of terrestrial foods such as bamboo shoots was likely underrepresented.

We investigated the composition of the forest via stratified random sampling, i.e., we subdivided the central part of the home range of the study group, which largely corresponds to the core area of the home range, into 5 distinct forest types or strata (Mueller-Dombois and Ellenberg 1974). We established a total of 67 plots of $20 \mathrm{~m} \times$ $20 \mathrm{~m}$ each (area: $26,800 \mathrm{~m}^{2}$ ) in which we recorded species, total height, bole height, crown diameter, and circumference for all trees $(n=1851)$ with girth $>40 \mathrm{~cm}$. The different strata and the exact vegetation sampling regime are described fully in Li et al. (2008).

On a monthly basis, we recorded presence/absence of fruits, flowers, and young leaves for 157 food trees and calculated the percentage of trees bearing each of the phenophases every month. For details on phenology monitoring, see Grueter et al. (2008a).

\section{Data Analysis}

One can obtain an indication of the degree to which primates are selective in their choice of food tree species by calculating a selection index (Krebs 1999). The index compares the proportion of feeding observations of a plant species with the relative abundance of the species concerned as estimated from the tree plots. We used basal area to express the relative species crown biomass and potential food abundance, and calculated the selection index, $W$, from the formula:

$$
W_{i}=O_{i} / P_{i}
$$

wherein $O_{i}$ is the percentage of feeding observations for species $i$, and $P_{i}$ is the percentage of total basal area accounted for by species $i . W_{i}>1$ indicates preference and $W_{i}<1$, avoidance.

Using tree data obtained from the plots, we measured the pattern of dispersion of important food tree species. We used the coefficient of dispersion (CD; $c f$. Koenig et al. 1998). The CD refers to the ratio of the variance to the mean of the number of species in a sample. If species were distributed randomly, their allocation across the plots in the sample would correspond to a Poisson distribution (mean equals variance; $C D=1) . C D>1$ indicates a clumped/contagious distribution, while a $C D<1$ shows a uniform distribution. We determined significant departures from randomness, i.e., departure from a variance-to-mean ratio of 1.0 , using the $\chi^{2}$ statistic (Brower et al. 1998; Perry and Mead 1979).

To examine if unit members avoided cofeeding, we tested whether OMU members had fewer nearest neighbors when feeding versus resting. To assess whether valued patches of food were being exhausted, we used opportunistic evidence of total patch depletion, i.e., leafy patches defoliated as a result of intensive foraging. 


\section{Results}

\section{Forest Composition}

We recorded a total of 80 tree species of 23 families in the botanical plots (Fig. 1; Table I). An additional 9 species of rare woody plants - Bretschneidera sinensis (Bretschneideraceae), Magnolia campbellii (Magnoliaceae), Corylus chinensis (Betulaceae), Populus yunnanensis (Salicaceae), Meliosma yunnanensis (Sabiaceae), Cerasus conadenia (Rosaceae), Clethra delavayi (Clethraceae), Ilex delavayi (Aquifoliaceae), and Rhododendron sinogrande (Ericaceae) - are not represented
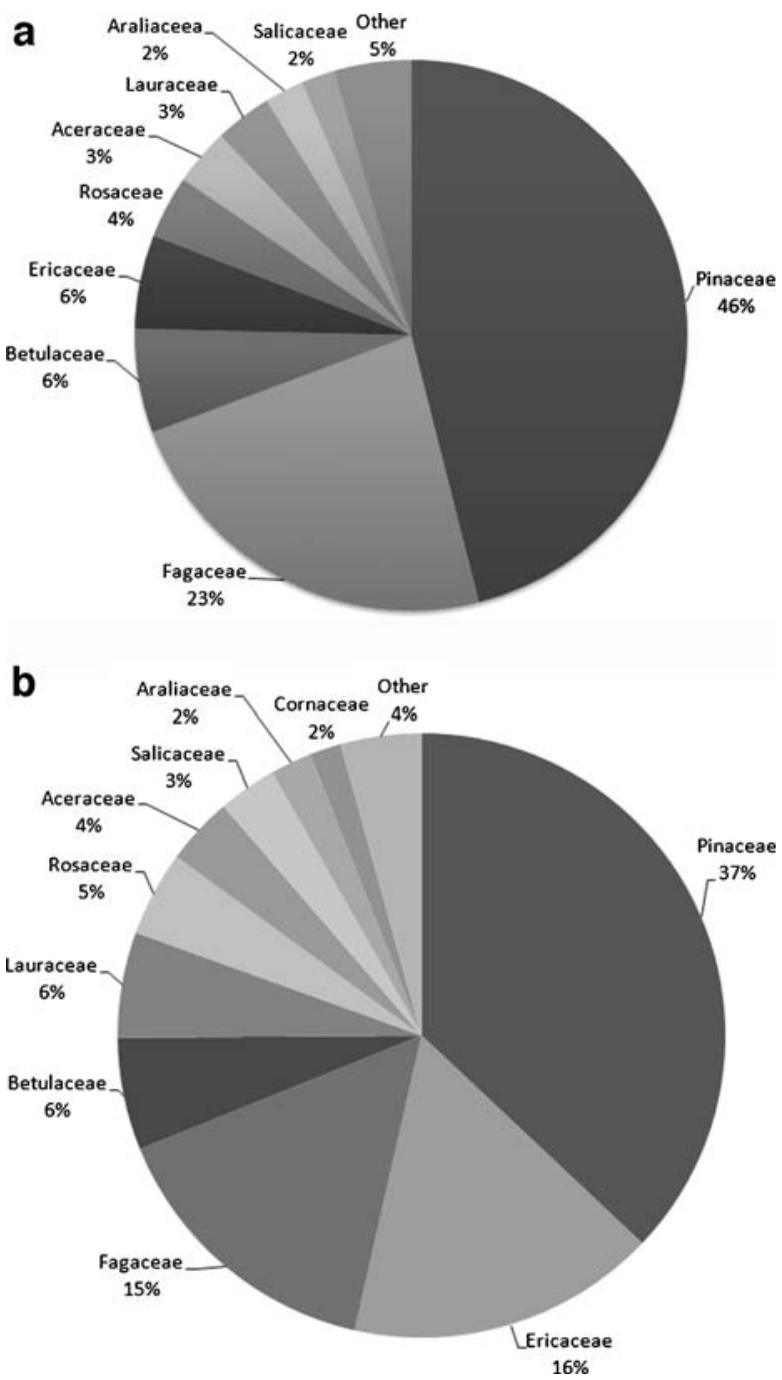

Fig. 1 The most common plant families at the Samage Forest, Baimaxueshan Nature Reserve. (a) Based on basal area. (b) Based on no. of stems $(n=1898)$. 
Table I The 20 most common tree species at the Samage Forest based on basal area

\begin{tabular}{lllll}
\hline Rank & Species & Family & Basal area/ha $\left(\mathrm{cm}^{2} / \mathrm{ha}\right)$ & $\%$ of total biomass \\
\hline 1 & Abies georgei & Pinaceae & 84,734 & 13.3 \\
2 & Cyclobalanopsis cf. gambleana & Fagaceae & 83,359 & 13.0 \\
3 & Picea likiangensis & Pinaceae & 80,014 & 12.5 \\
4 & Pinus yunnanensis & Pinaceae & 57,869 & 9.0 \\
5 & Tsuga dumosa & Pinaceae & 41,230 & 6.4 \\
6 & Quercus pannose & Fagaceae & 37,128 & 5.8 \\
7 & Abies ernestii & Pinaceae & 23,294 & 3.6 \\
8 & Rhododendron rubiginosum & Ericaceae & 18,651 & 2.9 \\
9 & Quercus rehderiana & Fagaceae & 18,185 & 2.8 \\
10 & Alnus nepalensis & Betulaceae & 15,389 & 2.4 \\
11 & Acanthopanax evodiaefolius & Araliaceae & 14,724 & 2.3 \\
12 & Betula utilis & Betulaceae & 14,644 & 2.3 \\
13 & Machilus microcarpa & Lauraceae & 14,333 & 2.2 \\
14 & Acer laxiflorum & Aceraceae & 10,015 & 1.6 \\
15 & Corylus cf. wangii & Betulaceae & 8802 & 1.4 \\
16 & Sorbus cf. thibetica & Rosaceae & 8245 & 1.3 \\
17 & Quercus aliena & Fagaceae & 8094 & 1.3 \\
18 & Taxus yunnanensis & Taxaceae & 7315 & 1.1 \\
19 & Tilia chinensis & Tiliaceae & 6288 & 1.0 \\
20 & Cornus macrophylla & Cornaceae & 6166 & 1.0 \\
\hline & & & & \\
\hline & & & & \\
& & & &
\end{tabular}

in the plots. The Pinaceae contributed the greatest biomass at Samage based on both basal area and stem density. The 2 top families together, Pinaceae and Fagaceae, accounted for $69 \%$ of the total basal area. The 3 top families, these 2 plus Ericaceae, together accounted for $75 \%$ of the total basal area and $69 \%$ of the total stem density. The 3 dominant tree species by basal area at Samage were Abies georgei, Cyclobalanopsis cf. gambleana, and Picea likiangensis. Thirty-five percent of the tree species were evergreen, and $65 \%$ were deciduous. Of the conifers $(n=10$ species), $10 \%$ were deciduous (Larix), whereas $27 \%$ of the broadleaf trees were evergreen.

\section{Dietary Repertoire}

Approximately 150 different vegetative food items from at least 94 species and 38 families contributed to the diet of the focal group. Foraging occurred both on the ground and in the canopy. Subjects obtained food items were obtained from 40 woody plant species (49\% of all available tree species), 22 shrubs, 1 semiparasitic shrub, 7 vines, 2 root-parasitic herbs, and 14 species of terrestrial herbaceous vegetation (THV). Food lists are provided in Tables II and III. In terms of stem density, food trees, excluding species supporting lichen only, accounted for $30.4 \%$ ( $n=40$ species) of the trees in the study area. In terms of relative dominance, the basal area of food trees comprised $35 \%$ of the total basal area in the study area. The 
Table II Food repertoire of Rhinopithecus bieti at Samage: vascular plants

\begin{tabular}{llll}
\hline $\begin{array}{l}\text { Predominant } \\
\text { life form }\end{array}$ & Species & Part & \\
\hline
\end{tabular}

\begin{tabular}{|c|c|c|c|}
\hline & Angiosperms & & \\
\hline & Fagaceae & & \\
\hline Tree & Quercus aliena & Shoot & Apr \\
\hline \multirow[t]{2}{*}{ Tree } & Quercus pannosa & Seed & Sep \\
\hline & & Flower & Jul \\
\hline \multirow[t]{3}{*}{ Tree } & Cyclobalanopsis cf. gambleana & Seed & Jan \\
\hline & & Pith & Apr \\
\hline & Juglandaceae & & \\
\hline \multirow[t]{2}{*}{ Tree } & Pterocarya delavayi & Young leaf & Apr \\
\hline & Aceraceae & & \\
\hline Tree & Acer sp. & Bud & Feb \\
\hline Tree & Acer sp. & Seed & Sep \\
\hline \multirow[t]{3}{*}{ Tree } & Acer laxiflorum var. laxiflorum & Young leaf & Apr, May \\
\hline & & Petiole & May \\
\hline & & Flower & May \\
\hline Tree & Acer mono var macropterum & Young leaf & Apr \\
\hline Tree & Acer caesium & Young leaf & Apr \\
\hline Tree & Acer hookeri & Young leaf & Apr \\
\hline \multirow[t]{3}{*}{ Tree } & Acer caudatum & Flower bud & May \\
\hline & & Young leaf & May \\
\hline & Bretschneideraceae & & \\
\hline \multirow[t]{3}{*}{ Tree } & Bretschneidera sinensis & Petiole & Oct \\
\hline & & Seed & Sep \\
\hline & Araliaceae & & \\
\hline \multirow[t]{7}{*}{ Tree } & Acanthopanax evodiaefolius & Mature leaf & Jul, Aug, Sep, Oct \\
\hline & & Young leaf & Apr, May, Jun \\
\hline & & Flower bud & Feb, Apr \\
\hline & & Fruit & $\begin{array}{l}\text { Aug, Sep, Oct, Nov, Jan, } \\
\text { Feb }\end{array}$ \\
\hline & & Bark & Mar, May, Sep \\
\hline & & Bud & Jan, Feb, Mar, Dec \\
\hline & & Petiole & Jun, Jul \\
\hline \multirow[t]{2}{*}{ Shrub } & $\begin{array}{l}\text { Acanthopanax leucorrhizus var. } \\
\text { fulvescens }\end{array}$ & Leaf & Oct \\
\hline & Celastraeae & & \\
\hline \multirow[t]{2}{*}{ Tree } & Euonymus theifolius & Seed & Oct \\
\hline & Hydrangeaceae & & \\
\hline \multirow[t]{2}{*}{ Tree } & Hydrangea heteromalla & Mature leaf & Jul, Sep, Oct, Nov \\
\hline & & Pith & Jul \\
\hline Tree & Hydrangea $\mathrm{sp}$. & Mature leaf & Aug \\
\hline Tree & Hydrangea $\mathrm{sp}$. & Bark & Jan \\
\hline
\end{tabular}


Table II (continued)

\begin{tabular}{llll}
\hline $\begin{array}{l}\text { Predominant } \\
\text { life form }\end{array}$ & Species & Part & Month \\
\hline
\end{tabular}

\begin{tabular}{|c|c|c|c|}
\hline Tree & Philadelphus delavayi var. delavayi & $\begin{array}{l}\text { Mature leaf } \\
\text { Bark }\end{array}$ & $\begin{array}{l}\text { Oct, Sep, Aug } \\
\text { Mar }\end{array}$ \\
\hline Shrub & Philadelphus sp. & Young leaf & May \\
\hline Shrub & Deutzia glomeruliflora & $\begin{array}{l}\text { Mature leaf } \\
\text { Young leaf } \\
\text { Flower }\end{array}$ & $\begin{array}{l}\text { Jul, Nov } \\
\text { May } \\
\text { May }\end{array}$ \\
\hline & Clethraceae & & \\
\hline Tree & Clethra delavayi & $\begin{array}{l}\text { Petiole } \\
\text { Young leaf }\end{array}$ & $\begin{array}{l}\text { May } \\
\text { May }\end{array}$ \\
\hline & Cornaceae & & \\
\hline Shrub & Helwingia japonica & Mature leaf & Sep \\
\hline Tree & Cornus macrophylla & $\begin{array}{l}\text { Fruit } \\
\text { Young leaf }\end{array}$ & $\begin{array}{l}\text { Aug, Sep } \\
\text { Apr }\end{array}$ \\
\hline & Rosaceae & & \\
\hline Tree & Sorbus rufopilosa & $\begin{array}{l}\text { Mature leaf } \\
\text { Fruit }\end{array}$ & $\begin{array}{l}\text { Jul, Oct } \\
\text { Aug, Sep }\end{array}$ \\
\hline Tree & Sorbus rehderiana var. cupreonitens & Young leaf & May \\
\hline Tree & Sorbus sp. & Young leaf & Jun \\
\hline Tree & Sorbus sp. & Bud & Feb \\
\hline Tree & Sorbus oligodonta & $\begin{array}{l}\text { Young leaf } \\
\text { Mature leaf } \\
\text { Fruit }\end{array}$ & $\begin{array}{l}\text { Apr } \\
\text { Oct, Nov } \\
\text { Aug, Oct, Nov }\end{array}$ \\
\hline Tree & Sorbus monbeigii & Mature leaf & Jul, Sep \\
\hline Tree & Sorbus hupehensis & Young leaf & Apr \\
\hline Tree & Sorbus macrantha & $\begin{array}{l}\text { Mature leaf } \\
\text { Fruit }\end{array}$ & $\begin{array}{l}\text { Oct } \\
\text { Jan, Oct }\end{array}$ \\
\hline Tree & Sorbus thibetica & $\begin{array}{l}\text { Bud } \\
\text { Young leaf } \\
\text { Fruit } \\
\text { Flower, flower } \\
\text { bud }\end{array}$ & $\begin{array}{l}\text { Feb } \\
\text { Apr, May, Jun } \\
\text { Aug, Sep, Oct, Nov } \\
\text { Apr, May }\end{array}$ \\
\hline Tree & Sorbus epidendron & Fruit & Feb \\
\hline Shrub & Stranvaesia davidiana & $\begin{array}{l}\text { Mature leaf } \\
\text { Fruit }\end{array}$ & $\begin{array}{l}\text { Mar, Apr, Dec } \\
\text { Oct }\end{array}$ \\
\hline Tree & Padus obtusata & $\begin{array}{l}\text { Fruit } \\
\text { Young leaf } \\
\text { Mature leaf } \\
\text { Bud } \\
\text { Flower bud }\end{array}$ & $\begin{array}{l}\text { Jun, Oct } \\
\text { Apr, May, Jun } \\
\text { Jul, Oct } \\
\text { Feb } \\
\text { May }\end{array}$ \\
\hline Tree & Malus yunnanensis & Young leaf & Apr, May \\
\hline Tree & Malus cf. prattii & Fruit & Sep \\
\hline
\end{tabular}


Table II (continued)

\begin{tabular}{|c|c|c|c|}
\hline $\begin{array}{l}\text { Predominant } \\
\text { life form }\end{array}$ & Species & Part & Month \\
\hline Shrub & Rosa macrophylla & Seed & Oct \\
\hline Shrub & Rubus alexeterius & Fruit & Jul \\
\hline Tree & Cerasus conadenia & Mature leaf & Oct \\
\hline \multirow[t]{2}{*}{ Tree } & Cerasus patentipila & Flower bud & May \\
\hline & & Young leaf & May \\
\hline \multirow[t]{3}{*}{ Tree } & Cerasus clarofolia & Young leaf & Apr \\
\hline & & Flower bud & Apr \\
\hline & Caprifoliaceae & & \\
\hline Shrub & Lonicera tangutica & Mature leaf & Oct \\
\hline Vine & Lonicera acuminata & Mature leaf, fruit & Mar \\
\hline Shrub & Viburnum betulifolium & Fruit & Feb, Oct \\
\hline Shrub & Viburnum nervosum & Young leaf & May \\
\hline Tree & Viburnum cylindricum & Fruit & Oct \\
\hline Herb & Sambucus adnata & Fruit & Sep \\
\hline \multirow[t]{2}{*}{ Shrub } & Leycesteria formosa & Mature leaf & Aug \\
\hline & Balanophoraceae & & \\
\hline \multirow{2}{*}{$\begin{array}{l}\text { Root-parasitic } \\
\text { herb }\end{array}$} & Balanophora involucrata & Tuber & Oct \\
\hline & Lililiaceae & & \\
\hline Shrub & Polygonatum cirrhifolium & Mature leaf & Aug \\
\hline Herb & Maianthemum atropurpureum & Leaf & May \\
\hline \multirow[t]{2}{*}{ Herb } & Maianthemum forrestii & All & Jun \\
\hline & Berberidaceae & & \\
\hline \multirow[t]{2}{*}{ Shrub } & Berberis sp. & Young leaf & Apr \\
\hline & Ranunculaeae & & \\
\hline Vine & Clematis obtusidentata & Mature leaf & Oct \\
\hline Vine & Clematis chrysocoma & Mature leaf & May \\
\hline \multirow[t]{2}{*}{ Herb } & Thalictrum delavayi & Leaf & Oct \\
\hline & Orobanchaceae & & \\
\hline \multirow{2}{*}{$\begin{array}{l}\text { Root-parasitic } \\
\text { herb }\end{array}$} & Boschniakia himalaica & Tuber & Nov \\
\hline & Balsaminaceae & & \\
\hline Herb & Impatiens arguta & Leaf & Oct \\
\hline \multirow[t]{2}{*}{ Herb } & Impatiens xanthocephala & Leaf & Oct \\
\hline & Uriticaceae & & \\
\hline Herb & Urtica sp. & Leaf & Oct \\
\hline Herb & Pilea angulata & Leaf & May, Oct, Nov \\
\hline \multirow[t]{2}{*}{ Herb } & Elatostema obtusum & Leaf & Nov \\
\hline & Cruciferae & & \\
\hline Herb & $\begin{array}{l}\text { Cardamine macrophylla var. } \\
\text { macrophylla }\end{array}$ & Leaf & Nov \\
\hline
\end{tabular}


Table II (continued)

\begin{tabular}{|c|c|c|c|}
\hline $\begin{array}{l}\text { Predominant } \\
\text { life form }\end{array}$ & Species & Part & Month \\
\hline Herb & $\begin{array}{l}\text { Eutrema yunnanense } \\
\text { Schisandraceae }\end{array}$ & Leaf & Nov \\
\hline Vine & Gramineae & $\begin{array}{l}\text { Fruit } \\
\text { Young leaf }\end{array}$ & $\begin{array}{l}\text { Nov } \\
\text { May }\end{array}$ \\
\hline Shrub & Fargesia cf. dura & $\begin{array}{l}\text { Leaf } \\
\text { Shoot }\end{array}$ & $\begin{array}{l}\text { Feb, Mar, Sep, Oct, Nov } \\
\text { Jun, Aug }\end{array}$ \\
\hline Shrub & Fargesia cf. melanostachys & $\begin{array}{l}\text { Young leaf } \\
\text { Mature leaf } \\
\text { Shoot }\end{array}$ & $\begin{array}{l}\text { Mar, Apr, May } \\
\text { Jul, Nov, Dec } \\
\text { May, Jun, Jul }\end{array}$ \\
\hline Shrub & Fargesia sp. & Mature leaf & Jan, Aug \\
\hline Shrub & $\begin{array}{l}\text { Yushania sp. } \\
\text { Tiliaceae }\end{array}$ & Mature leaf & Oct \\
\hline Tree & Salicaceae & $\begin{array}{l}\text { Mature leaf } \\
\text { Young leaf }\end{array}$ & $\begin{array}{l}\text { Oct } \\
\text { Apr }\end{array}$ \\
\hline Tree & Salix rehderiana & $\begin{array}{l}\text { Young leaf } \\
\text { Catkin }\end{array}$ & $\begin{array}{l}\text { Apr } \\
\text { Mar, Apr }\end{array}$ \\
\hline Tree & Populus davidiana & $\begin{array}{l}\text { Young leaf } \\
\text { Flower bud } \\
\text { Bud } \\
\text { Bark }\end{array}$ & $\begin{array}{l}\text { Apr } \\
\text { Mar } \\
\text { Mar } \\
\text { Feb, Oct }\end{array}$ \\
\hline & Betulaceae & & \\
\hline Tree & Betula utilis & Young leaf & May \\
\hline Tree & Unid. species & Young leaf & Apr \\
\hline Tree & $\begin{array}{l}\text { Corylus ferox } \\
\text { Lauraceae }\end{array}$ & Young leaf & Apr \\
\hline Tree & Machilus yunnanensis & $\begin{array}{l}\text { Bud } \\
\text { Mature leaf }\end{array}$ & $\begin{array}{l}\text { Mar } \\
\text { Apr, Sep }\end{array}$ \\
\hline Tree & Machilus microcarpa & Seed & Sep \\
\hline Tree & Litsea chunii & $\begin{array}{l}\text { Young leaf } \\
\text { Flower }\end{array}$ & $\begin{array}{l}\text { Apr, May } \\
\text { Apr }\end{array}$ \\
\hline & Oleaceae & & \\
\hline Shrub & Ligustrum cf. delavayanum & Fruit & Oct \\
\hline Tree & $\begin{array}{l}\text { Syringa yunnanensis } \\
\text { Leguminosae }\end{array}$ & Mature leaf & Aug \\
\hline Shrub & $\begin{array}{l}\text { Piptanthus nepalensis } \\
\text { Compositae }\end{array}$ & Fruit & Sep \\
\hline Herb & Senecio scandens & Leaf & Oct \\
\hline Herb & Ligularia nelumbifolia & Leaf & Aug \\
\hline
\end{tabular}


Table II (continued)

\begin{tabular}{|c|c|c|c|}
\hline $\begin{array}{l}\text { Predominant } \\
\text { life form }\end{array}$ & Species & Part & Month \\
\hline Tree & Rhododendron rubiginosum & Flower & May \\
\hline Shrub & $\begin{array}{l}\text { Rhododendron yunnanense } \\
\text { Rhododendron selense }\end{array}$ & $\begin{array}{l}\text { Young leaf } \\
\text { Petiole }\end{array}$ & $\begin{array}{l}\text { May, Jun } \\
\text { May }\end{array}$ \\
\hline Tree & Enkianthus cf. deflexus & Young leaf & Apr \\
\hline Shrub & Enkianthus chinensis & Flower & Apr \\
\hline Shrub & $\begin{array}{l}\text { Lyonia villosa } \\
\text { Loranthaceae }\end{array}$ & Young leaf & Apr \\
\hline $\begin{array}{l}\text { Semi-parasitic } \\
\text { shrub }\end{array}$ & Laradizabalaceae & All & Oct \\
\hline Vine & Holboellia angustifolia & Mature leaf & Apr \\
\hline Shrub & $\begin{array}{l}\text { Decaisnea fargesii } \\
\text { Acrinidiaceae }\end{array}$ & Fruit & Aug \\
\hline Vine & Actinidia pilosula & $\begin{array}{l}\text { Young leaf } \\
\text { Fruit }\end{array}$ & $\begin{array}{l}\text { Apr } \\
\text { Oct }\end{array}$ \\
\hline & Vitaceae & & \\
\hline Vine & $\begin{array}{l}\text { Cayratia cf. cardiospermoides } \\
\text { Sabiaceae }\end{array}$ & Fruit & Oct \\
\hline Tree & $\begin{array}{l}\text { Meliosma yunnanensis } \\
\text { Saxifragaceae }\end{array}$ & Fruit, mature leaf & Oct \\
\hline Herb & $\begin{array}{l}\text { Chrysoplenium davidianum } \\
\text { Aquifoliaceae }\end{array}$ & All & \\
\hline Tree & Ilex sp. & $\begin{array}{l}\text { Mature leaf } \\
\text { Flower } \\
\text { Young leaf }\end{array}$ & $\begin{array}{l}\text { Jan, Feb, Apr, Nov, Dec } \\
\text { Dec } \\
\text { Feb }\end{array}$ \\
\hline Tree & Ilex delavayi & $\begin{array}{l}\text { Mature leaf } \\
\text { Bark }\end{array}$ & $\begin{array}{l}\text { Jul, Aug } \\
\text { Jan }\end{array}$ \\
\hline & $\begin{array}{l}\text { Gymnopserms } \\
\text { Cupressaeae }\end{array}$ & & \\
\hline Tree & $\begin{array}{l}\text { Sabina squamata } \\
\text { Pinaceae }\end{array}$ & Fruit & Mar \\
\hline Tree & Larix speciosa & Petiole & Apr, May, Jul, Sep \\
\hline
\end{tabular}

The list is based on systematic scan observations, opportunistic observations, and food remnants.

top 10 food tree species (Table IV) accounted for $>90 \%$ of the total feeding time on plant foods. All top 10 food tree species had selection ratios $>1$, with a few species having extraordinarily high selection indices, i.e., Pterocarya delavayi (71.7; represented by a single specimen in the plots), Padus obtusata (41.8), and Acanthopanax evodiaefolius (20.4; Table IV). Many species listed in Table II were fed on infrequently. As estimated from feeding records, immature leaves of Acanthopanax evodiaefolius were the single most prominent food type (Table V). 
Table III Food repertoire of Rhinopithecus bieti at Samage: cryptogams, i.e., nonvascular plants (lichens) and fungi

\begin{tabular}{|c|c|}
\hline Species & Season \\
\hline \multicolumn{2}{|l|}{ Lichens } \\
\hline \multicolumn{2}{|l|}{ Parmeliaceae $^{\mathrm{a}}$} \\
\hline Usnea longissima & Year-round \\
\hline Usnea sp. & Year-round \\
\hline Bryoria confusa & Year-round \\
\hline Bryoria $\mathrm{cf}$ trichodes cf. ssp. americana & Year-round \\
\hline Cetrelia sp. & Apr, Dec \\
\hline 1 unk species & Nov \\
\hline \multicolumn{2}{|l|}{ Fungi } \\
\hline \multicolumn{2}{|l|}{ Russulaceae } \\
\hline Russula sp. 1 & Jul, Aug, Sep, Oct \\
\hline Russula sp. 2 & Sep \\
\hline Russula sp. 3 & Aug \\
\hline Russula senecis & Sep \\
\hline \multicolumn{2}{|l|}{ Amanitaceae } \\
\hline Amanita hemibapha var. ochracea & Aug \\
\hline Amanita fritillaria & Sep \\
\hline Amanita flavipes & Sep \\
\hline \multicolumn{2}{|l|}{ Gomphaceae } \\
\hline Gomphus floccosus & Aug, Sep \\
\hline \multicolumn{2}{|l|}{ Boletaceae } \\
\hline Boletus sp. & Aug, Sep \\
\hline
\end{tabular}

The list is based on systematic scan observations, opportunistic observations, and food remnants.

${ }^{a}$ The species of lichens have previously been reported in Grueter et al. (2009).

As estimated from feeding remains, shoots of Fargesia spp. were the most important dietary item, followed by fruits of Acanthopanax evodiaefolius, fruits of Sorbus cf. thibetica, fruits of Sorbus spp., and mature leaves of A. evodiaefolius. The average number of plant species and specific plant food items used per month was 16 and 19, respectively. The richness of food species (controlled for observation time) peaked in April/May, August, and October (Fig. 2).

Mature leaves were chosen and ingested from both deciduous and evergreen trees, but only a few woody species were important sources of mature leaves, i.e., the deciduous Philadelphus delavayi, Sorbus spp., Acanthopanax evodiaefolius, and the evergreen Ilex sp. For some species, only petioles were eaten, e.g., Bretschneidera sinensis; for others, only the leaf blades, e.g., Stranvaesia davidiana; and for yet others both leaf blades and petioles, e.g., Acanthopanax evodiaefolius.

The snub-nosed monkeys fed on subterranean parts of Boschniakia himalaica and Balanophora involucrata. They spent a considerable amount of time unearthing unidentified tubers (hidden food items). Juveniles and females ate snow in winter, but only on rare occasions. The monkeys drank water from small ponds and streams. 
Table IV Percentage of feeding records ${ }^{\mathrm{a}}$ for the 10 top-ranked identified plant species (out of all records of plant feeding for which the plant species could be identified to at least genus level)

\begin{tabular}{lllll}
\hline Rank & Species & Family & \% of feeding records & Selection index \\
\hline 1 & Acanthopanax evodiaefolius & Araliaceae & 47.0 & 20.4 \\
2 & Sorbus spp. $^{\text {b }}$ & Rosaceae & 15.3 & 9.5 \\
3 & Acer spp. $^{\text {c }}$ & Aceraceae & 7.7 & 3.4 \\
4 & Sorbus cf. thibetica & Rosaceae & 6.3 & 4.9 \\
5 & Fargesia spp. ${ }^{\text {d }}$ & Gramineae & 5.5 & NA $^{\mathrm{e}}$ \\
6 & Pterocarya delavayi & Juglandaceae & 4.5 & 71.7 \\
7 & Cornus macrophylla & Cornaceae & 1.8 & 1.8 \\
8 & Padus obtusata & Rosaceae & 1.5 & 41.8 \\
9 & Tilia cf. chinensis & Tiliaceae & 1.2 & 1.2 \\
10 & Litsea chunii & Lauraceae & 0.9 & 8.6 \\
\hline
\end{tabular}

\footnotetext{
${ }^{1}$ In total, there were 2674 identified feeding records (1788 for lichens, 886 for plants).

${ }^{\mathrm{b}}$ Includes Sorbus oligodonta, S. rufopilosa, S. rehderiana, S. monbeigii, S. hupehensis, and S. macrantha; distinguishing among these species was difficult.

${ }^{\mathrm{c}}$ Includes Acer laxiflorum, A. mono. A. hookeri, A. caesium, and A. caudatum; distinguishing among these species was difficult.

${ }^{\mathrm{d}}$ Includes Fargesia cf. melanostachys and F. cf. dura. Identifying bamboos to species level was not possible.
}

e Bamboo.

Table V Number of feeding records for the 10 top-ranked specific food items

\begin{tabular}{|c|c|c|c|}
\hline Rank & Item & Family & No. of feeding records \\
\hline 1 & Acanthopanax evodiaefolius (young leaves) & Araliaceae & 207 \\
\hline 2 & Acanthopanax evodiaefolius (fruits) & Araliaceae & 174 \\
\hline 3 & Sorbus spp. ${ }^{\text {a }}$ (fruits) & Rosaceae & 107 \\
\hline 4 & Acer spp. ${ }^{\mathrm{b}}$ (young leaves) & Aceraceae & 63 \\
\hline 5 & Fargesia spp. $^{\mathrm{c}}$ (mature leaves) & Gramineae & 49 \\
\hline 6 & Pterocarya delavayi (young leaves) & Juglandaceae & 42 \\
\hline 7 & Acanthopanax evodiaefolius (buds) & Araliaceae & 40 \\
\hline 8 & Sorbus cf. thibetica (young leaves) & Rosaceae & 35 \\
\hline 9 & Sorbus cf. thibetica (fruits) & Rosaceae & 19 \\
\hline 10 & Cornus macrophylla (fruits) & Cornaceae & 17 \\
\hline
\end{tabular}

\footnotetext{
${ }^{a}$ ncludes Sorbus oligodonta, S. rufopilosa, S. rehderiana, S. monbeigii, S. hupehensis, and S. macrantha; distinguishing among these species was difficult.

${ }^{\mathrm{b}}$ Includes Acer laxiflorum, A. mono. A. hookeri, A. caesium, and A. caudatum; distinguishing among these species was difficult.

${ }^{\mathrm{c}}$ Includes Fargesia cf. melanostachys and F. cf. dura. Identifying bamboos to species level was not possible.
} 
Contrary to Xiang et al. (2007), we never observed the monkeys of this population eating resin. We recorded a case of predation on bird eggs. One individual fed on the flesh of an unidentified flying squirrel (Sciuridae). We observed the monkeys biting into mushrooms in the fall. We also saw them removing the bark of dead fallen and standing trees, mostly Abies georgei, and disassembling rotten and brittle tree stumps. Although we never clearly saw an individual actually eating an insect, the latter observations may indicate foraging on invertebrates. We witnessed feeding on bamboo (Fargesia spp.) leaves in all seasons. The monkeys consumed large quantities of bamboo shoots (Fargesia spp.) in summer.

\section{Food Competition}

Although the overall density of trees was 708 stems/ha, the 3 main food trees comprised only 42.5 individuals/ha (6.0\%; Table VI). The single most important woody food species, Acanthopanax evodiaefolius, occurred in only $16.4 \%$ of the vegetation plots. All top 3 food tree taxa-Acanthopanax evodiaefolius, Sorbus thibetica, and Sorbus spp.- were clumped in distribution. On several occasions, we encountered evergreen trees that were completely defoliated after the group of Rhinopithecus bieti had visited them, demonstrating full patch depletion (Table VII). The monkeys especially sought the leaves of Ilex and Philadelphus. OMU members had fewer nearest neighbors when feeding versus resting. When an OMU member was feeding ( $n=5020$; excluding bachelors and infants), there was another member in proximity ( $0-2$ arm's lengths) in only $4.6 \%$ of all records versus $41.5 \%$ when an individual was resting.

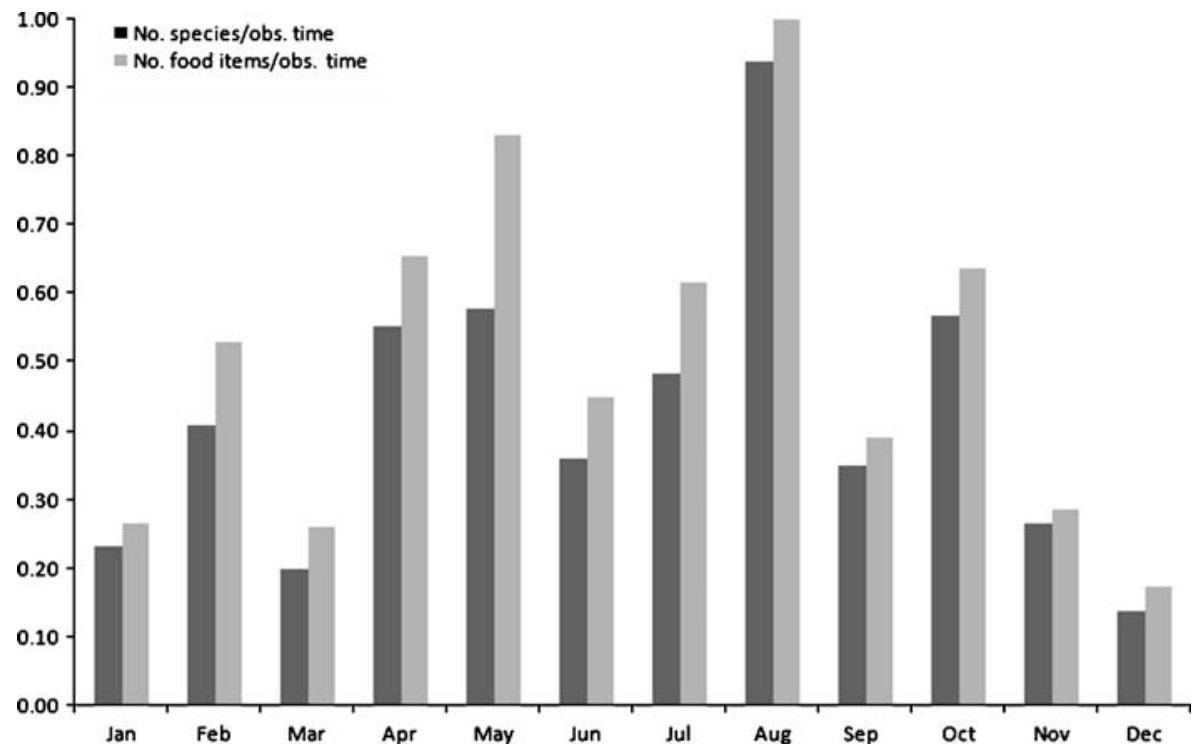

Fig. 2 Food species richness of Rhinopithecus bieti at Samage compared among months, calculated as the number of food species and food items per observation time (scan-based visual observation). 
Table VI Density and dispersion of major ${ }^{a}$ food of Rhinopithecus bieti at Samage

\begin{tabular}{llll}
\hline Species & $n$ & $\begin{array}{l}\text { Density } \% \text { plots }^{\mathrm{c}} \\
\left(\mathrm{ha}^{-1}\right)\end{array}$ & $\begin{array}{l}\text { Index of dispersion Distribution } \\
\text { (variance-to- } \\
\text { mean-ratio) }\end{array}$
\end{tabular}

\begin{tabular}{lllllll}
\hline Acanthopanax evodiaefolius & 41 & 15.3 & $16.4(31.4)$ & $5.2 * *\left(4.7^{* *}\right)$ & Clumped & 3.7 \\
Sorbus thibetica & 16 & 6.0 & $17.9(28.6)$ & $1.4 * *(1.3)$ & Clumped $^{\text {d }}$ & 1.3 \\
Sorbus spp. & 57 & 21.3 & $26.9(48.6)$ & $3.8^{* *}\left(3.1^{* *}\right)$ & Clumped & 3.1 \\
A. evodiaefolius, & 114 & 42.5 & $40.3(68.6)$ & $5.7 * *\left(4.5^{* *}\right)$ & Clumped & 4.2 \\
$\begin{array}{l}\text { S. thibetica \& Sorbus spp. } \\
\text { All }\end{array}$ & 1898 & 708.2 & & & & \\
\hline
\end{tabular}

a "Major" here refers to the 2 most important food plant genera Sorbus and Acanthopanax, which together make up almost $69 \%$ of the feeding records.

${ }^{\mathrm{b}}$ Includes Sorbus oligodonta, S. rufopilosa, S. rehderiana, S. monbeigii, S. hupehensis, and S. macrantha; these species were lumped together for the analyses because distinguishing among them in situ was not straightforward.

${ }^{\mathrm{c}}$ Percentage of plots with species $i$.

${ }^{\mathrm{d}}$ Random in mixed forest.

${ }^{* *}$ Designates a significant deviation from randomness $(p<0.05)$. Numbers in parentheses refer to the 35 plots in mixed forest only.

\section{Discussion}

We demonstrated that the dietary richness of this population of Rhinopithecus bieti encompassed 94 different plant species and a few unusual items: mammals, mushrooms, tubers, bamboo. Moreover, we showed that plant species were not consumed in accordance with their spatial abundance, but that the monkeys showed a strong preference for uncommon species with a clumped distribution and that, based on several lines of evidence, they may experience some scramble and contest competition.

\section{Plant Food Selection and Diversity}

Seventy-five percent of the woody stems at Samage were Pinaceae (pines, firs, hemlocks and spruces), Fagaceae (oaks), and Ericaceae (rhododendrons), none of

Table VII Ad libitum observations of broadleaf trees having been depleted through intense foraging by Rhinopithecus bieti

\begin{tabular}{llc}
\hline Species & No. of depleted trees and dates & BA (\%) \\
\hline Philadelphus delavayi & 5 (Oct 05), 2 (Nov 05), 5 (Aug 06), 2 (Oct 06), 2 (Nov 06) & 0.08 \\
Ilex sp. & 2 (Oct 05), 1 (Jan 07) & 0.03 \\
Ilex delavayi & 3 (Oct 05), 3 (Nov 05), 1 (Feb 06), 1 (Mar 06), 2 (Aug 06), & $0^{\mathrm{a}}$ \\
Hydrangea heteromalla & 2 (Oct 06), 4 (Nov 06) & 0.03 \\
Malus yunnanensis & 1 (July 07) & 0.09 \\
\hline
\end{tabular}

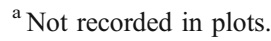


which were of direct dietary importance to the snub-nosed monkeys ( $c f$. Kirkpatrick 1996). However, conifers and oaks were important lichen-bearing trees and were almost exclusively exploited for this nonplant resource (Grueter et al. 2009). Rhinopithecus bieti at Samage derived its plant diet mostly from the deciduous angiosperms families Araliaceae, Rosaceae, Aceraceae, Juglandaceae, and Cornaceae. High selection ratios for most of these angiosperm plant species indicate strong selectivity for uncommon species, which is likely due to variability in nutritional quality.

Only a few fruit species provided the majority of the diet in summer and fall, viz. Acanthopanax evodiaefolius, Sorbus spp., Sorbus cf. thibetica, and Cornus macrophylla. Of Sorbus and Acanthopanax, the monkeys ate both fruits and mature leaves at the same time, often in an alternating fashion. Mature leaves contributed relatively marginally to the diet of $R$. bieti at Samage (4\%; Grueter et al. 2009). Most of the ingested mature leaves (most notably A. evodiaefolius, Sorbus spp., Padus obtusata, Hydrangea heteromalla, Philadelphus delavayi, Fargesia spp., and Ilex spp.) were deciduous with the exception of Ilex spp. and Fargesia sp. Sayers and Norconk (2008) demonstrated a similar preference for broad-leaved deciduous mature leaves over evergreen mature leaves in Himalayan langurs. The digestibility of short-lived deciduous leaves is almost universally superior to that of the evergreen leaves (Coley 1988). Rhinopithecus bieti at Samage also displayed a preference for young over mature leaves (Grueter et al. 2009). Compared to mature foliage, young foliage generally has higher nutritional quality; it is higher in protein, lower in fiber and secondary compounds (Boonratana 1993; McKey et al. 1981; Milton 1979; Oates et al. 1980).

\section{Intra- and Interspecific Differences}

There has been some discrepancy with regard to the typical feeding strategy of Rhinopithecus bieti. Kirkpatrick (1996) stressed the species' relatively monotonous dependence on lichens (specialist) whereas Ding and Zhao (2004) accentuated its dietary diversity (generalist). This inconsistency is likely a consequence of different habitat characteristics. Populations of Rhinopithecus bieti occur in different ecological conditions, and findings from the Wuyapiya population, which inhabits one extreme of the habitat of $R$. bieti (Kirkpatrick 1996), are not representative of all other populations. In a gradient from south to north, precipitation and temperature decrease while average altitude of occupied habitat increases and the vegetation becomes progressively poorer. The limited diet of the Wuyapiya and also Xiaochangdu population (Xiang et al. 2007) in the north is a consequence of low species richness. The Samage forest is floristically richer and more productive than Xiaochangdu and Wuyapiya and foraging options are thus less constrained. Our study confirms that the dietary spectrum and key foods largely depend on the particular habitat, climate condition, botanical composition, and species richness. The number of species consumed clearly decreases with increasing altitude and latitude. At the northern end of the geographical range of Rhinopithecus bieti (Wuyapiya and Xiaochangdu), they consumed 20 and 25 plant species in 12 and 13 mo, respectively (Kirkpatrick 1996; Xiang et al. 2007). As for the central part of the species' range, the group at Tacheng-Xiagguqing fed upon 50 plant species over 14 
mo of study while the group at Samage-Gehuaqing ate 75 plant species over the first 14 mo of study and 94 species over the total of 20 mo (Ding and Zhao 2004; this study). At the southern end (Longma), they incorporated an assumed 97 species into the diet (data derived from indirect evidence such as trail signs only; Huo 2005). Diet breadth (species richness) of the Samage and Longma populations is comparable to that of most tropical sites where researchers have studied Asian colobines (Curtin 1980; Davies 1991; Kool 1993).

The top ranking dietary genus of Rhinopithecus bieti at Samage was Acanthopanax (alternatively named Eleutherococcus), of which the monkeys consumed all major phytophases, viz. young leaves in spring; mature leaves in summer and fall; fruits in summer, fall, and winter; buds in winter; and bark all year round. Acanthopanax was not part of the diet of Rhinopithecus bieti at Wuyapiya and Xiaochangdu, probably due to the genus' low density there (Kirkpatrick 1996), but it is harvested by populations of $R$. bieti living to the south of Samage (Liu et al. 2004). The closely related allopatric and ecologically comparable Rhinopithecus roxellana has a similar diet, both in terms of food plant genera and species (Guo et al. 2007; Li 2006). Among the non-Rhinopithecus taxa, Himalayan langurs of Nepal are of great comparative value owing to their association with biogeographically, botanically, and topographically similar habitats. While Acanthopanax is a key food plant species for Rhinopithecus bieti, Himalayan langurs did not include Acanthopanax at all into the diet despite the genus being relatively common there (Sayers and Norconk 2008). Conversely, the Himalayan langurs ate the shrub Gaultheria in considerable quantities, and despite being superabundant at Samage, evidence for inclusion in the diet of Himalayan snub-nosed monkeys is lacking.

\section{What Do These Data Tell Us About the Possibility of Food Competition?}

We demonstrated for this species and population that preferred food trees were spatially clumped, occurred at low densities across the home range, and were possibly not large enough to accommodate a fairly cohesive 400-member band at the same time. The distributional features are a prerequisite for contest competition (Koenig et al. 1998; van Schaik 1989; cf. Isbell et al. 1998). Researchers have so far not addressed for this population food quality, which also impacts the competitive potential (van Schaik 1989).

Some comparatively rare and highly preferred, and supposedly high-quality, plant resource patches, e.g., leaves of Ilex and Philadelphus, became depleted after the group of Rhinopithecus bieti had encountered them. Along the same lines, Kirkpatrick (1996) noted that $\geq 3$ Sorbus trees were defoliated in the path of the band of Rhinopithecus bieti. Ilex spp. (holly) and Philadelphus delavayi (mock orange) occurred at very low stem densities, and also in terms of basal area, they all accounted for only $0.15 \%$ of the total basal area of all trees. These opportunistic data indicate that Rhinopithecus bieti undeniably depleted some plant resources, although full depletion was mostly restricted to a few scarce species.

Some authors have proposed that an efficient way to alleviate the costs of food competition is to avoid cofeeding (Saj and Sicotte 2007b; Siex and Struhsaker 1999; van Noordwijk and van Schaik 1987) by spreading out while feeding and consequently having fewer neighbors while feeding versus resting. Our results 
confirm that feeding individuals had fewer conspecifics in proximity than resting ones. However, we never observed a female behaving competitively toward other female unit members while feeding in a patch or supplanting another female from a food source, behaviors that would indicate the presence of contest competition.

While competition within units was perhaps more of the scramble type, the low density and clumped distribution of preferred resources, e.g., fruiting trees, waterholes, are suggestive of interunit contest competition. We previously showed that male aggressive behavior correlates positively with temporal availability of fruit (Grueter 2009), which, however, could have been confounded by some other factor. Further, the only recorded band fission event occurred in late winter when valued fruit resources became rare and extremely patchy (Grueter et al. 2008a, b). Ad libitum observations substantiate the possibility of contest competition among units: in May 2006, large, dominant OMUs appeared to defend leafing trees (rare Pterocarya trees) from other nearby units. The lower ranking units appeared to wait in nearby conifer trees eating lichens until the more dominant units left the leafing trees. In January 2007, 1 unit chased away another unit from an Acanthopanax tree that still bore fruits. These observations recall Isbell's (1991) proposition that species that feed on both dispersed and clumped resources might reduce competition by shifting from clumped to dispersed foods. Researchers have also reported competition among social units for access to food trees/feeding areas for Rhinopithecus roxellana (Zhang et al. 2003), Colobus guereza (Harris 2006), and C. polykomos (Korstjens 2001).

Although still preliminary, our results demonstrate the pervasiveness of moderate food competition in Rhinopithecus bieti, at least with regard to nonlichen foods (plants). We would expect this to have a constraining effect on the species' socioecology, i.e., limit group size. However, groups are unusually large, which implies that either feeding competition is not severe enough to constrain group size, or that there is an advantage of forming large groups that counteracts the disadvantage associated with feeding competition, such as threats stemming from nearby roaming all-male units (Grueter 2009; Grueter et al. 2008b).

\section{Conclusion and Areas for Future Research}

Owing to earlier observations suggesting that Rhinopithecus bieti feed almost exclusively on lichens, researchers originally characterized the species as having one of the most specialized diets of all primates. Subsequent studies including this one have confirmed that lichens are indeed a key dietary component, but also underpinned the dietary elasticity of Rhinopithecus bieti in response to variation in availability, abundance, and diversity of plant food supply. Rhinopithecus bieti at Samage exhibit a rather broad usage of the resources in their habitat ( $>90$ plant species) and thus can be viewed as generalists. However, the dominant evergreen plant families did not offer many palatable foodstuffs to the monkeys, which instead relied heavily on a few rather uncommon deciduous hardwood species. Some highly sought food trees occurred at very low densities and were irregularly distributed in space and time, which are preconditions for the emergence of contest competition. In line with recent studies, our study provides preliminary evidence consistent with the recent contention that feeding competition may be more widespread among 
colobines than previously thought. Future studies should aim to obtain a better understanding of food competition in the species, for instance by gathering data on patch residence time, patch size, and unit size to evaluate patch depletion, a measurable behavioral indicator of the presence or absence of intragroup scramble competition (Snaith and Chapman 2005).

A salient finding of our study is that some mature leaves were totally avoided by Rhinopithecus bieti whereas others were highly sought after. It is widely known that protein content and fiber have a strong influence on leaf choice in colobines (Davies et al. 1988; Fashing et al. 2007; Oates et al. 1980; Waterman and Choo 1981). Future research should investigate the phytochemical components associated with preferred and avoided food items, thereby contributing to a fuller comprehension of the feeding ecology of Rhinopithecus bieti.

Acknowledgments Mr. Sikang Liu granted permission to work at Baimaxueshan National Nature Reserve. This work would have been impossible without our trackers Shunkai Feng, Xuesheng Feng, and Xuewen Feng. We thank Hai Xian, Liu Jingxin, Fang Zhendong, and Xiao Maorong at the Alpine Botanical Garden in Shangri-La; Sun Hang at the Herbaria of the Kunming Institute of Botany (KIB); and Yang Yuming (The Nature Conservancy) for help with plant specimen identifications; Yang Zhuliang (KIB) for identification of fungi specimens; and Wang Lisong (KIB) for identification of lichen specimens. C. C. Grueter thanks Huo Sheng, Xiao Wen, Quan Ruichang, and Xiang Zuofu at the Kunming Institute of Zoology (KIZ) for constant hospitality and fruitful discussions. We also thank Ken Sayers and the anonymous reviewers for instructive comments on the content of the manuscript and proofreading of the text. This study was made possible by grants from Janggen-Pöhn-Stiftung, A. H. Schultz Stiftung, Zürcher Tierschutz, G. \& A. Claraz-Schenkung, Goethe-Stiftung, Jane Goodall Institute Schweiz, and the Kommission für Reisestipendien der Schweizerischen Akademie der Naturwissenschaften SANW (all in Switzerland), and the Offield Family Foundation, Primate Conservation, Inc., Zoological Society of San Diego, and the Primate Action Fund of Conservation International (all in the United States). The research was noninvasive and abided by the legal requirements in the People's Republic of China.

\section{References}

Anderson, D. P., Nordheim, E. V., Boesch, C., \& Moermond, T. C. (2002). Factors influencing fissionfusion grouping in chimpanzees in the Taï National Park, Côte d'Ivoire. In C. Boesch, G. Hohmann \& L. F. Marchant (Eds.), Behavioural diversity in chimpanzees and bonobos (pp. 90-101). Cambridge, UK: Cambridge University Press.

Barton, R. A., \& Whiten, A. (1994). Reducing complex diets to simple rules: Food selection by olive baboons. Behavioral Ecology and Sociobiology, 35, 283-293.

Bauchop, T., \& Martucci, R. W. (1968). Ruminant-like digestion of the langur monkey. Science, 161, 698-700.

Bennett, E. L., \& Davies, A. G. (1994). The ecology of Asian colobines. In A. G. Davies \& J. F. Oates (Eds.), Colobine monkeys: Their ecology, behaviour and evolution (pp. 129-171). Cambridge, UK: Cambridge University Press.

Bleisch, W., Cheng, A.-S., Ren, X.-D., \& Xie, J.-H. (1993). Preliminary results from a field study of wild Guizhou snub-nosed monkeys (Rhinopithecus brelichi). Folia Primatologica, 60, 72-82.

Boonratana, R. (1993). The ecology and behaviour of the proboscis monkey (Nasalis larvatus) in the Lower Kinabatangan. Ph.D. dissertation: Mahidol University, Thailand.

Brower, J. E., Zar, J. H., \& von Ende, C. N. (1998). Field and laboratory methods for general ecology. Boston: WCB/McGraw-Hill.

Caton, J. M. (1998). The morphology of the gastrointestinal tract of Pygathrix nemaeus (Linnaeus, 1771). In N. G. Jablonski (Ed.), The natural history of the doucs and snub-nosed monkeys (pp. 129-152). Singapore: World Scientific Press.

Chapman, C. A. (1990). Ecological constraints on group size in three species of neotropical primates. Folia Primatologica, 73, 1-9. 
Chapman, C. A., \& Chapman, L. J. (2000). Determinants of group size in primates: the importance of travel costs. In S. Boinski \& P. A. Garber (Eds.), On the move: How and why animals travel in groups (pp. 24-42). Chicago: University of Chicago Press.

Coley, P. D. (1988). Effects of plant growth rate and leaf lifetime on the amount and type of anti-herbivore defense. Oecologia, 74, 531-536.

Curtin, S. H. (1980). Dusky and banded leaf monkeys. In D. J. Chivers (Ed.), Malayan forest primates: Ten years' study in tropical rain forest (pp. 107-145). New York: Plenum Press.

Dasilva, G. L. (1994). Diet of Colobus polykomos on Tiwai Island: Selection of food in relation to its seasonal abundance and nutritional quality. International Journal of Primatology, 15, 655-680.

Davies, A. G. (1991). Seed-eating by red leaf monkeys (Presbytis rubicunda) in dipterocarp forest of northern Borneo. International Journal of Primatology, 12, 119-144.

Davies, A. G., Bennett, E. L., \& Waterman, P. G. (1988). Food selection by two south-east Asian colobine monkeys (Presbytis rubicunda and Presbytis melalophos) in relation to plant chemistry. Biological Journal of the Linnean Society, 34, 33-56.

Ding, W., \& Zhao, Q. K. (2004). Rhinopithecus bieti at Tacheng, Yunnan: Diet and daytime activities. International Journal of Primatology, 25, 583-598.

Fashing, P. J. (2001a). Activity and ranging patterns of guerezas in the Kakamega Forest: Intergroup variation and implications for intragroup feeding competition. International Journal of Primatology, 22, 549-577.

Fashing, P. J. (2001b). Feeding ecology of guerezas in the Kakamega Forest, Kenya: The importance of Moraceae fruit in their diet. International Journal of Primatology, 22, 579-606.

Fashing, P. J. (2007). African colobine monkeys: Patterns of between-group interaction. In C. J. Campbell, A. Fuentes, K. C. MacKinnon, M. Panger \& S. K. Bearder (Eds.), Primates in perspective (pp. 201224). Oxford: Oxford University Press.

Fashing, P. J., Dierenfeld, E. S., \& Mowry, C. B. (2007). Influence of plant and soil chemistry on food selection, ranging patterns, and biomass of Colobus guereza in Kakamega Forest, Kenya. International Journal of Primatology, 28, 673-703.

Garber, P. A. (1987). Foraging strategies among living primates. Annual Review of Anthropology, 16, 339-364.

Gillespie, T. R., \& Chapman, C. A. (2001). Determinants of group size in the red colobus monkey (Procolobus badius): An evaluation of the generality of the ecological-constraints model. Behavioral Ecology and Sociobiology, 50, 329-338.

Grueter, C. C. (2009). Determinants of modular societies in snub-nosed monkeys (Rhinopithecus bieti) and other Asian colobines, Ph.D. thesis, University of Zurich, Zurich, Switzerland.

Grueter, C. C., \& Xiang, Z.-F. (2008). Fallback foods and primate adaptability: Primates in temperate environments. American Journal of Physical Anthropology, Suppl, 46, 109.

Grueter, C. C., Li, D., van Schaik, C. P., Ren, B., Long, Y., \& Wei, F. (2008a). Ranging of Rhinopithecus bieti in the Samage Forest, China. I. Characteristics of range use. International Journal of Primatology, 29, 1121-1145.

Grueter, C. C., van Schaik, C. P., Ren, B., \& Wei, F. (2008b). Towards an understanding of the social system of black-and-white snub-nosed monkeys (Rhinopithecus bieti). Primate Eye, 96, 317.

Grueter, C. C., Li, D., Ren, B, Wei, F., Xiang, Z., van Schaik, C. P. (2009). Fallback foods of temperateliving primates: A case study on snub-nosed monkeys. American Journal of Physical Anthropology.

Guo, S., Li, B., \& Watanabe, K. (2007). Diet and activity budget of Rhinopithecus roxellana in the Qinling Mountains, China. Primates, 48, 268-276.

Harris, T. (2006). Between-group contest competition for food in a highly folivorous population of black and white colobus monkeys (Colobus guereza). Behavioral Ecology and Sociobiology, 61, 317-329.

Hume, I. D. (1989). Optimal digestive strategies in mammalian herbivores. Physiological Zoology, 62, $1145-1163$.

Huo, S. (2005). Diet and habitat use of Rhinopithecus bieti at Mt Longma. Kunming Institute of Zoology, Kunming, China: Yunnan. Ph.D. dissertation.

Isbell, L. A. (1991). Contest and scramble competition: Patterns of female aggression and ranging behaviour among primates. Behavioral Ecology, 2, 143-155.

Isbell, L. A., Pruetz, J. D., \& Young, T. P. (1998). Movements of vervets (Cercopithecus aethiops) and patas monkeys (Erythrocebus patas) as estimators of food resource size, density, and distribution. Behavioral Ecology and Sociobiology, 42, 123-133.

Janson, C. H., \& Goldsmith, M. L. (1995). Predicting group size in primates: Foraging costs and predation risks. Behavioral Ecology, 6, 326-336.

Janson, C. H., \& van Schaik, C. P. (1988). Recognizing the many faces of primate food competition: Methods. Behaviour, 105, 165-186. 
Kay, R. F. (1984). On the use of anatomical features to infer foraging behavior in extinct primates. In P. S. Rodman \& J. G. H. Cant (Eds.), Adaptations for foraging in nonhuman primates: Contributions to an organismal biology of prosimians, monkeys, and apes (pp. 21-53). New York: Columbia University Press.

Kay, R. N. B., \& Davies, A. G. (1994). Digestive physiology. In A. G. Davies \& J. F. Oates (Eds.), Colobine monkeys: Their ecology, behaviour and evolution (pp. 229-249). Cambridge: Cambridge University Press.

Kirkpatrick, R. C. (1996). Ecology and behavior of the Yunnan snub-nosed langur (Rhinopithecus bieti, Colobinae), Ph.D. thesis, University of California, Davis.

Kirkpatrick, R. C. (2007). The Asian colobines: Diversity among leaf-eating monkeys. In C. J. Campbell, A. Fuentes, K. C. MacKinnon, M. Panger \& S. K. Bearder (Eds.), Primates in perspective (pp. 186200). Oxford: Oxford University Press.

Koenig, A., Beise, J., Chalise, M. K., \& Ganzhorn, J. U. (1998). When females should contest for food: Testing hypotheses about resource density, distribution, size, and quality with Hanuman langurs (Presbytis entellus). Behavioral Ecology and Sociobiology, 42, 225-237.

Koenig, A., \& Borries, C. (2001). Socioecology of Hanuman langurs: The story of their success. Evolutionary Anthropology, 10, 122-137.

Koenig, A., McCullough, E., \& Borries, C. (2008). Patch depletion, satiation, and patch occupancy: Ecological constraints in Phayre's leaf monkeys (Trachypithecus phayrei). American Journal of Physical Anthropology, Suppl, 46, 133.

Kool, K. M. (1993). The diet and feeding behavior of the silver leaf monkey (Trachypithecus auratus sondaicus) in Indonesia. International Journal of Primatology, 14, 667-700.

Korstjens, A. H. (2001). The mob, the secret sorority, and the phantoms, Ph.D. thesis, Utrecht University, Utrecht.

Krebs, C. J. (1999). Ecological methodology. Menlo Park, CA: Addison-Wesley-Longman.

Kuhn, J. H. (1964). Zur Kenntnis von Bau und Funktion des Magen der Schlankaffen. Folia Primatologica, 2, 193-221.

Lambert, J. E. (2007). Primate nutritional ecology: Feeding biology and diet at ecological and evolutionary scales. In C. J. Campbell, A. Fuentes, K. C. MacKinnon, M. Panger \& S. K. Bearder (Eds.), Primates in perspective (pp. 482-495). Oxford: Oxford University Press.

Li, Y. (2006). Seasonal variation of diet and food availability in a group of Sichuan snub-nosed monkeys in Shennongjia Nature Reserve, China. American Journal of Primatology, 68, 217-233.

Li, D., Grueter, C. C., Ren, B., Long, Y., Li, M., Peng, Z., et al. (2008). Ranging of Rhinopithecus bieti in the Samage Forest, China. II. Use of land cover types and altitudes. International Journal of Primatology, 29, 1147-1173.

Litvaitis, J. A. (2000). Investigating food habits of terrestrial vertebrates. In L. Boitani \& T. K. Fuller (Eds.), Research techniques in animal ecology (pp. 165-190). New York: Columbia University Press.

Liu, Z., Ding, W., \& Grüter, C. C. (2004). Seasonal variation in ranging patterns of Yunnan snub-nosed monkeys Rhinopithecus bieti a Mt. Fuhe, China. Acta Zoologica Sinica, 50, 691-696.

Majolo, B., de Bortoli Vizioli, A., \& Schino, G. (2008). Costs and benefits of group living in primates: Group size effects on behaviour and ecology. Animal Behaviour, 76, 1235-1247.

McKey, D. B., Gartlan, J. S., Waterman, P. G., \& Choo, G. M. (1981). Food selection by black colobus monkeys (Colobus satanas) in relation to plant chemistry. Biological Journal of the Linnean Society, 16, 115-146.

Milton, K. (1979). Factors influencing leaf choice by howler monkeys: A test of some hypotheses of food selection by generalist herbivores. American Naturalist, 114, 362-378.

Milton, K. (1981). Food choice and digestive strategies of two sympatric primate species. American Naturalist, 117, 496-505.

Milton, K. (1984). The role of food-processing factors in primate food choice. In P. S. Rodman \& J. G. H. Cant (Eds.), Adaptations for foraging in nonhuman primates: Contributions to an organismal biology of prosimians, monkeys, and apes (pp. 249-279). New York: Columbia University Press.

Mueller-Dombois, D., \& Ellenberg, H. (1974). Aims and methods of vegetation ecology. New York: John Wiley \& Sons.

Oates, J. F. (1987). Food distribution and foraging behavior. In B. B. Smuts, D. L. Cheney, R. N. Seyfarth, R. W. Wrangham \& T. T. Struhsaker (Eds.), Primate societies (pp. 197-209). Chicago: University of Chicago Press.

Oates, J. F. (1994). The natural history of African colobines. In A. G. Davies \& J. F. Oates (Eds.), Colobine monkeys: Their ecology, behaviour and evolution (pp. 75-128). Cambridge: Cambridge University Press.

Oates, J. F., Waterman, P. G., \& Choo, G. M. (1980). Food selection in the South Indian leaf-monkey, Presbytis johnii, in relation to leaf chemistry. Oecologia, 45, 45-56. 
Oftedal, O. T. (1991). The nutritional consequences of foraging in primates: The relationship of nutrient intakes to nutrient requirements. Philosophical Transactions of the Royal Society of London B, 334, $161-170$.

Perry, J. N., \& Mead, R. (1979). On the power of the index of dispersion test to detect spatial pattern. Biometrics, 35, 613-622.

Saj, T. L., \& Sicotte, P. (2007a). Predicting the competitive regime of female Colobus vellerosus from the distribution of food resources. International Journal of Primatology, 28, 315-336.

Saj, T. L., \& Sicotte, P. (2007b). Scramble competition among Colobus vellerosus at Boabeng-Fiema, Ghana. International Journal of Primatology, 28, 337-355.

Sayers, K., \& Norconk, M. (2008). Himalayan Semnopithecus entellus at Langtang National Park, Nepal: Diet, activity patterns, and resources. International Journal of Primatology, 29, 509-530.

Siex, K. S., \& Struhsaker, T. T. (1999). Ecology of the Zanzibar red colobus monkey: Demographic variability and habitat stability. International Journal of Primatology, 20, 163-192.

Snaith, T. V., \& Chapman, C. A. (2005). Towards an ecological solution to the folivore paradox: Patch depletion as an indicator of within-group scramble competition in red colobus monkeys (Piliocolobus tephrosceles). Behavioral Ecology and Sociobiology, 59, 185-190.

Snaith, T. V., \& Chapman, C. A. (2008). Red colobus monkeys display alternative behavioral responses to the costs of scramble competition. Behavioral Ecology, 19, 1289-1296.

Steenbeek, R., \& van Schaik, C. P. (2001). Competition and group size in Thomas's langurs (Presbytis thomasi): The folivore paradox revisited. Behavioral Ecology and Sociobiology, 49, 100-110.

Sterck, E. H. M., Watts, D. P., \& van Schaik, C. P. (1997). The evolution of female social relationships in nonhuman primates. Behavioral Ecology and Sociobiology, 41, 291-309.

Stevens, C. E. (1988). Comparative physiology of the vertebrate digestive system. Cambridge: Cambridge University Press.

Struhsaker, T. T. (1975). The red colobus monkey. Chicago: University of Chicago Press.

Struhsaker, T. T., \& Oates, J. F. (1975). Comparison of the behavior and ecology of red colobus and blackand-white colobus monkeys in Uganda: A summary. In R. H. Tuttle (Ed.), Socioecology and psychology of primates (pp. 103-123). The Hague: Mouton Publishers.

Teichroeb, J. A., Saj, T. L., Paterson, J. D., \& Sicotte, P. (2003). Effect of group size on activity budgets of Colobus vellerosus in Ghana. International Journal of Primatology, 24, 743-758.

van Noordwijk, M. A., \& van Schaik, C. P. (1987). Competition among female long-tailed macaques, Macaca fascicularis. Animal Behaviour, 35, 577-589.

van Schaik, C. P. (1989). The ecology of social relationships amongst female primates. In V. Standen \& R. A. Foley (Eds.), Comparative socioecology (pp. 195-218). Oxford: Blackwell.

van Schaik, C. P., \& van Noordwijk, M. A. (1988). Scramble and contest in feeding competition among female long-tailed macaques (Macaca fascicularis). Behaviour, 105, 77-98.

Waterman, P. G., \& Choo, G. M. (1981). The effects of digestibility-reducing compounds in leaves on food selection of some Colobinae. Malaysian Applied Biology, 10, 147-162.

Wrangham, R. W. (1980). An ecological model of female bonded primate groups. Behaviour, 75, 262300.

Xiang, Z.-F., Huo, S., Xiao, W., Quan, R.-C., \& Grueter, C. C. (2007). Diet and feeding behavior of Rhinopithecus bieti at Xiaochangdu, Tibet: Adaptations to a marginal environment. American Journal of Primatology, 69, 1141-1158.

Yang, S.-J., \& Zhao, Q.-K. (2001). Bamboo leaf-based diet of Rhinopithecus bieti at Lijiang, China. Folia Primatologica, 72, 92-95.

Yeager, C. P., \& Kirkpatrick, R. C. (1998). Asian colobine social structure: Ecological and evolutionary constraints. Primates, 39, 147-155.

Yeager, C. P., \& Kool, K. (2000). The behavioral ecology of Asian colobines. In P. F. Whitehead \& C. F. Jolly (Eds.), Old World monkeys (pp. 497-521). Cambridge: Cambridge University Press.

Zhang, P., Li, B.-G., Wada, K., Tan, C. L., \& Watanabe, K. (2003). Social structure of Sichuan snub-nosed monkeys (Rhinopithecus roxellana) in the Qinling Mountains of China. Acta Zoologica Sinica, 49, 727-735. 